\title{
Zur soziologischen Auseinandersetzung mit Gewalt in der Medizin am Beispiel des medizinischen Kinderschutzes
}

\section{Christoph Sucherdt}

(C) Der/die Autor(en) 2020

Zusammenfassung Der medizinische Kinderschutz ist ein durch die gegenwärtige deutsche Soziologie kaum beachtetes Phänomen. Der Artikel möchte auf der einen Seite spezifische Entwicklungen des medizinischen Kinderschutzes aufzeigen. Hierzu wird aus einer wissenssoziologischen Perspektive insbesondere auf seine Entstehung, Entwicklung der Definitionen von Misshandlung, die Diagnose verschiedener Formen von Gewalt und auf die Rolle der medizinischen Forschung eingegangen. Auf der anderen Seite sollen besondere Dynamiken und Folgen, welche die Beschäftigung der Medizin mit dem Gegenstand der Gewalt gegen Kinder mit sich bringen, eingehender soziologisch betrachtet werden.

Schlüsselwörter Medizinischer Kinderschutz · Kindesmisshandlung · Medizinsoziologie · Gewaltsoziologie

C. Sucherdt $(\bowtie)$

Fachbereich 01, Institut für Sozialwesen, Universität Kassel, Arnold-Bode-Straße 10, 34127 Kassel, Deutschland

E-Mail: christoph.sucherdt@uni-kassel.de 


\title{
A medical approach toward violence sociological revisited: the case of medical child protection
}

\begin{abstract}
Medical child protection is a phenomenon receiving little attention from contemporary German sociology. The article begins to look at specific topics related to medical child protection such as the development of this medical field, the development of the definition of child abuse, the diagnostic of different forms of child abuse and some aspects of medical research regarding this topic. Later on the article tries to gain a deeper sociological gaze of some dynamics and consequences resulting of the engagement of medicine with the phenomenon of violence against children.
\end{abstract}

Keywords Medical child protection $\cdot$ Child abuse $\cdot$ Sociology of health and medicine $\cdot$ Sociology of violence

\section{Einleitung}

Der medizinische Kinderschutz ist eine sehr einflussreiche sozio-technische Praxis, die sich mit dem Problem der Gewalt gegen Kinder auseinandersetzt. In der gegenwärtigen deutschen Soziologie scheint dieses Thema - mit wenigen Ausnahmen (Alberth et al. 2014; Bode und Turba 2014; Franzheld 2017) - kaum Beachtung zu finden. Der medizinische Kinderschutz erscheint in deutschen soziologischen Debatten vielmehr randständig. Nach der Entstehung des medizinischen Kinderschutzes in den 1960er Jahren wurde er in der englischsprachigen Soziologie, z. B. als sozial konstruiertes Problem (Gelles 1976; Pfohl 1977) oder als Beispiel von Medikalisierung (Conrad und Schneider 1980) wie auch in der Wissenschaftsforschung (Hacking 1991, 1999) thematisiert. Inzwischen scheint er weitestgehend aus dem soziologischen Blick verschwunden zu sein. Wie verschiedene Studien zum Kinderschutz (Pfohl 1977; Hildenbrand 2014; Marks et al. 2018) zeigen, besitzt die medizinische Expertise im Umgang mit Gewalt eine nicht umgehbare Autorität im Feld. Weiterhin ist der medizinische Kinderschutz ein sehr dynamisches Phänomen, welches sich beständig weiterentwickelt. Eine - zumindest in Deutschland - aktuelle Dynamik, welche z.B. in Bezug auf die Frage nach der Organisation professioneller Arbeit (Klatetzki und Tacke 2005) interessant ist, ist etwa die hinsichtlich der Etablierung von multiprofessionellen Kinderschutzgruppen in Krankenhäusern zur Behandlung und Beurteilung von Verdachtsfällen (Thun-Hohenstein 2006; Inthorn 2012). In diesem Beitrag möchte ich zeigen, dass eine Auseinandersetzung mit dem medizinischen Kinderschutz für eine Soziologie der Gewalt lohnenswert sein kann, da es diesem Bereich innerhalb der Medizin gelungen ist, über das Problem der Gewalt gegen Kinder Definitionen von Gewalt sowie Praktiken der Gewalterkennung zu etablieren und darüber hinaus diese Konzeptionen gegenüber anderen Disziplinen durchzusetzen. Die medizinische Kinderschutzpraxis ist zentral daran beteiligt, zu bestimmen, ob es sich in einem fraglichen Fall um Gewalt handelt und dafür hat sie diverse diagnostische Instrumentarien entwickelt. 
Im Folgenden möchte ich zuerst auf die Genese des medizinischen Kinderschutzes eingehen und Besonderheiten dieser Entwicklung aufzeigen, welche sich als Medikalisierung verdichten lassen könnte. Danach werde ich die Entfaltung der medizinischen Definition von Gewalt und Kindesmisshandlung - von einem engen zu einem breiten Verständnis von Gefährdung - nachzeichnen. Anschließend sollen einige Aspekte der Diagnose von Gewalt im medizinischen Kontext thematisiert werden, wobei ein Augenmerk auf spezifische Komplexitätssteigerungen, die mit einer Misshandlungsdiagnose einhergehen, gelegt wird. Auf die Rolle der medizinischen Forschung zur Steigerung der Komplexität zur Begründung einer entsprechenden Diagnose wird im nächsten Abschnitt eingegangen. Abschließend sollen die wichtigsten Argumentationslinien des Textes zusammengefasst und in relevante soziologische Diskurse eingeordnet werden.

\section{Zur Entstehung des medizinischen Kinderschutzes}

Auch wenn es in einer Übersicht nach Hermann et al. (2016, S. $13 \mathrm{ff}$.) schon um 900 n. Chr. erste Wahrnehmungen von Gewalt gegen Kinder in der Medizin gab, ist mit dem Vortrag ,The Battered Child Syndrome“ des Pädiaters Henry Kempe auf der Jahrestagung der American Academy of Pediatrics im Jahr 1961 ein Ausgangspunkt der aktuellen Debatten zu identifizieren. Dieser Vortrag, 1962 von einem Autorenteam um Kempe als Text veröffentlicht (Kempe et al. 1962), wird von Hermann et al. (2016, S. 14) als epochemachend charakterisiert.

Vor dem Zeitpunkt der Etablierung des medizinischen Kinderschutzes hatten sich schon andere Disziplinen, wie die Soziale Arbeit (Lynch 1985), mit dem Thema auseinandergesetzt. Die Besonderheit des Ansatzes von Kempe et al. (1962) lag darin, dass sie Kindesmissbrauch als ein genuin medizinisches Phänomen definierten, welches nicht nur die Verletzungen des betroffenen Kindes, sondern auch die misshandelnden Bezugspersonen als psychologisch beeinträchtigt umfasste und damit gleichzeitig eine weitergehende Diskussion - die weit über medizinische Fachkreise hinausging - anstießen. Mit diesem „Auftakt“ verbreitete sich die Wahrnehmung von problematischen Kindesmisshandlungen über die USA hinaus.

Aber welche Bedingungen machten Kempe und den Begriff ,,battered child syndrome" so erfolgreich? Welche Faktoren führten zu der sog. Medikalisierung des Kinderschutzes (Görgen et al. 2013; Conrad und Schneider 1980)? Ein wichtiger Faktor ist schon die Bezeichnung des Phänomens als „,battered child syndrome“ gegenüber anderen Bezeichnungen wie etwa ,physical abuse syndrome“. Die Wahl des Namens war eine bewusste Entscheidung, um in der eigenen Disziplin und darüber hinaus mehr Aufmerksamkeit für das Thema zu erhalten. Nach den Aussagen seiner Tochter ist der emotionale Titel von Kempe „tailored to make the most impact“ (Kempe 2013, S. 11). Nicht nur der bildliche und emotionale Titel stieß auf fruchtbaren Boden, auch die Bezeichnung des Phänomens mit dem Begriff „Syndrom“ soll die Bereitschaft innerhalb der Medizin erhöht haben, den Misshandlungsdiskurs als ein für die Medizin relevantes Thema anzuerkennen (Görgen et al. 2013, S. 223). Wie Pfohl (1977, S. 315 ff..) herausstellt, gab es durchaus fachspezifische Barrieren innerhalb der Medizin, welche eine umfassende Anerkennung des The- 
mas behinderten. Denn in der medizinischen Praxis, wie z.B. in Notaufnahmen, wurden durchaus Verletzungen behandelt, die durch Gewaltausübungen der Eltern verursacht wurden. Aber sie wurden dort nicht als Gewaltverletzungen, sondern als „normale“ Verletzungen, unabhängig von ihren Ursachen behandelt (vgl. Conrad und Schneider 1980; Hacking 1991). Pfohl (1977) zufolge ermöglichte die Benennung des Problems als ein Syndrom, dass sich das Konzept innerhalb der Medizin durchsetzen konnte, obwohl es z.B. in Konflikt zum Vertrauensverhältnis zwischen Ärzt*innen und Patient*innen und dem medizinischen Interesse an der Kontrolle der Konsequenzen der eigenen Diagnosen steht.

Das für die (Fach-)Öffentlichkeit zurechtgeschnittene Thema stieß in dieser Zeit, aufgrund einer wachsenden Sensibilität gegenüber Gewalt in den USA (Williams 1983), auf große Resonanz. Weiterhin waren für die „Entdeckung“ zwei weitere Entwicklungen innerhalb der Medizin bedeutsam. Einen bedeutenden Anteil an ,einer erstmaligen Definition von Kindesmisshandlung als eigene[m] Symptomkomplex" (Görgen et al. 2013, S. 223) hatte die Entwicklung besserer diagnostischer Methoden, insbesondere der bildgebenden Radiologie, durch welche Spuren von Verletzungen sichtbar gemacht werden konnten, die zuvor kaum aufgedeckt werden konnten. ${ }^{1}$ Durch die technisch vermittelte Herstellung der Röntgenbilder suggerierten sie ,den Eindruck der Unabhängigkeit und der unbestechlichen Objektivität, die den vergleichsweise subjektiv erscheinenden Aussagen z. B. der Eltern gegenübergestellt werden konnten“" (Görgen et al. 2013, S. 223). Die radiologischen Bilder sind noch in einer weiteren Hinsicht relevant, denn sie ermöglichten medizinisch Forschenden, diagnostische Kategorien zur Aufdeckung von Misshandlungen zu entwickeln, ohne einen direkten Kontakt zu den Patient*innen haben zu müssen. Die radiologischen Aufnahmen konnten mit einer größeren emotionalen Distanz zu den Eltern und Kindern studiert werden, als dies z. B. Pädiater*innen normalerweise haben. Sie konnten ohne Handlungsdruck zur Behandlung der Patient*innen analysiert werden. Vor diesem Hintergrund ist es Pfohl (1977) zufolge kein Zufall, dass das Thema der Kindesmisshandlung zuerst von der pädiatrischen Radiologie „entdeckt“ wurde. Eingebettet war der Prozess der „Entdeckung“ in das Setting des Krankenhauses. Es bot Kempe und seinem Team eine kritische Masse an Patient*innen, um ähnliche Fälle zu bündeln und daraus typische Verletzungsmuster und radiologische Indikationen abzuleiten. Mit diesem Wissen und der radiologischen Methode wurde es ermöglicht, Fälle von Misshandlungen frühzeitiger zu identifizieren (Görgen et al. 2013, S. 223).

Mit der Medikalisierung des Kinderschutzes war eine Art unterschwelliges Versprechen verbunden, dass es durch medizinische Diagnosen möglich ist, Fälle von Kindesmisshandlung eindeutig und objektiv zu identifizieren und dass den Mediziner*innen eine besondere Verantwortung obliegt, sich dieses Problems (führend) anzunehmen. Bei Helfer (1978, S. 69f.), einem der frühen medizinischen Pioniere (zusammen mit Kempe), heißt es: „Es ist die Verantwortung des Arztberufes, die von uns die Führung auf diesem Gebiet verlangt“. Auch wenn dieses Versprechen auf

\footnotetext{
1 Die Bedeutung der radiologischen Bilder zeigt sich auch in dem Aufsatz von Kempe et al. (1962) zum „battered-child syndrome“, denn er ist ausschließlich mit radiologischen Aufnahmen bebildert, um die ,,verborgenen“ Verletzungsmuster aufzuzeigen.
} 
eindeutige Objektivität, z.B. von der medizinischen Forschung auf diesem Gebiet, unterlaufen wurde, scheint es immer noch fortzuleben.

Der durch andere Disziplinen anerkannte Deutungsanspruch der Medizin auf diesem Gebiet soll anhand von zwei Beispielen ausgeführt werden. Instruktiv für die ärztliche Perspektive ist z.B. ein Artikel von Verocai et al. (2013), in dem es um die Evaluation der Wirkung von Meldungen einer klinischen Kinderschutzgruppe an die Jugendwohlfahrt (JWF) in Österreich geht. Die Evaluation ergab, dass sich die Meldung von Verdachtsfällen der klinischen Kinderschutzgruppe positiv auf die Arbeit der Jugendwohlfahrt auswirke, weil die Gruppe der JWF neue Fälle zuführt und die ärztliche Diagnose eher zu einer Annahme von Hilfe bei den Eltern führe als die Diagnose der Sozialarbeiter*innen. Hier wird die Diagnose der klinischen Kinderschutzgruppe als ,ärztliche (Verdachts)-diagnose“ der ,Vermutungsdiagnose eines Mitarbeiters der JWF gegenübergestellt, die als Unterstellung abgetan werden kann" (Verocai et al. 2013, S. 237). Durch die Einklammerung des Verdachts bei der Attribuierung der ärztlichen Diagnose und die Charakterisierung der von Sozialarbeiter*innen vorgenommenen Diagnose als eine bloße Vermutung, die einfach in Zweifel gezogen werden könne, wird deutlich, welche Methode von Verocai et al. (2013) als objektiver angesehen wird. Die Argumentation lässt sich wie folgt zusammenfassen: Die medizinische Diagnose ist objektiv, ihr lässt sich (kaum) widersprechen und deshalb sei diese wirksamer als eine sozialarbeiterische Diagnose. Das korreliert mit Befunden über die Bearbeitung von Kinderschutzfällen in Jugendämtern. Bei Hildenbrand (2014, S. 184) heißt es dazu z. B.: „Offenbar unbelehrbar ist der sozialpädagogische Glaube an die ,Objektivität" medizinischer Befunde“. Die sozialpädagogischen Fachkräfte würden demzufolge dazu neigen, ihre eigene fachliche Perspektive zugunsten der Einschätzungen anderer Professionen, wie der Medizin oder auch der Psychologie, zu suspendieren (Marks et al. 2018, S. 13). Deshalb wird der Kinderschutz als ein medikalisiertes Feld beschrieben, in dem die Medizin eine relativ große Deutungshoheit etabliert hat (Conrad und Schneider 1980; Görgen et al. 2013; Pfohl 1977).

In Deutschland ist der medizinische Kinderschutz hingegen (noch) nicht so weitreichend etabliert wie in den USA. Nach einer Übersicht von Hermann et al. (2016, S. 16ff.) begann die Beschäftigung mit diesem Thema in Deutschland in den 1980er Jahren und verlief zunächst eher schleppend. Inzwischen scheint der medizinische Kinderschutz relativ fest in Deutschland verankert zu sein und sich weiter zu institutionalisieren. Dies zeigen zum einen Bestrebungen, Kinderschutzmedizin als eigenständiges Fach und als Bestandteil der Ausbildung von Pädiater*innen zu etablieren und zum anderen die ansteigende Zahl von Kinderschutzgruppen und Gewaltambulanzen in medizinischen Einrichtungen (Hermann et al. 2016, S. $16 \mathrm{ff}$.). In Österreich sind z. B. seit 2004 Kinderschutzgruppen an Kinderkliniken gesetzlich vorgeschrieben ( $\$ 8$ e des Bundesgesetztes über Kranken- und Kuranstalten) und nach Thun-Hohenstein (2016, S. 119) wurde diese Vorgabe auch ,flächendeckend umgesetzt"“. 


\section{Gewalt und Misshandlung im medizinischen Kinderschutz}

Im folgenden Abschnitt soll zuerst auf die Entwicklung der Definition von Misshandlung eingegangen werden. Der Begriff wurde schrittweise von einem relativ engen zu einem sehr umfassenden Gewaltbegriff ausgeweitet. Danach soll auf Besonderheiten von medizinischen Praktiken der Gewalterkennung - also auf die Diagnose von Gewalt und Misshandlung - eingegangen werden. Anschließend sollen Unterschiede zwischen den verschiedenen Formen von Missbrauch thematisiert werden.

\subsection{Zur Definition von Misshandlung und Gewalt}

$\mathrm{Zu}$ Beginn des medizinischen Kinderschutzes zielte die Definition von Kindesmissbrauch und damit das, was in der Praxis diagnostiziert werden sollte, hauptsächlich auf eher schwere physische Misshandlungen, wie schon die Benennung des Phänomens von Kempe et al. (1962) mit dem Begriff ,battered child syndrome“ aufzeigt. Das in Frage stehende Phänomen war bei Kempe et al. (1962) in ihrem ersten Aufsatz eng an spezifische Krankheitsbilder gekoppelt, wie z. B. an Knochenbrüche, welche durch Röntgenaufnahmen diagnostiziert werden können (Gelles 1976, S. 135f.). Noch bis in die 1970er Jahre beschäftigte sich die Medizin hauptsächlich mit dem Problem der körperlichen Misshandlung von Kindern (Söhner et al. 2017, S. 65). Im Laufe der Zeit wurde das Konzept der Kindesmisshandlung ausgeweitet und auf weitere Phänomene bezogen. Zu verweisen ist hier z. B. auf den Aufsatz vom Kempe (1978) mit dem Titel „Sexual Abuse, Another Hidden Pediatric Problem“. Bei Görgen et al. (2013, S. 223f.) wird die semantische Erweiterung des Begriffs child abuse als ein Teil der „Entgrenzung der medizinischen Einflusssphäre“ gedeutet. Die Entgrenzung erfolgte nach ihrer Darstellung in mehreren Schritten. Zunächst wurde unter child abuse nur (intentionale) körperliche Gewalt begriffen. Später wurde dem Konzept das elterliche Fehlverhalten hinzugefügt. Misshandlung war nicht mehr ausschließlich direkte Gewalt gegen Kinder, sondern auch die Behinderung einer optimalen kindlichen Entwicklung und die Vernachlässigung der kindlichen Bedürfnisse. In einem weiteren Schritt wurde der Begriff um eine sexualisierte Dimension ausgedehnt.

Verbunden mit der Dynamik der Entgrenzung ist ein Wechsel in der Terminologie des Gegenstandes. In dem am weitesten verbreiteten medizinischen Handbuch in Deutschland zum Thema heißt es dazu: ,Der Begriff ,Battered child' skizziert begrifflich nur eine umschriebene Befundkonstellation, die dem heute anerkannten, weiten Spektrum misshandlungsbedingter Befunde nicht mehr gerecht wird“ (Hermann et al. 2016, S. 24). Vorgeschlagen werden deshalb Begriffe wie ,nichtakzidentelle Verletzung“, , abusive injury“ oder ,,inflicted injury“ (Hermann et al. 2016, S. 24). Der zunächst eher spezifische und emotionale Begriff „,battered child syndrome“ wird also zugunsten eines neutraleren Begriffs mit größerer Reichweite abgelöst, indem der ursprüngliche Begriff als ein Unterphänomen davon eingebettet wird. Heutzutage wird i.d.R. zwischen physischer Misshandlung, sexuellem Missbrauch, Vernachlässigung und psychischer Misshandlung unterschieden.

Mit der semantischen Erweiterung des Begriffes child abuse verschärfte sich das Problem zu bestimmen, was Misshandlungen genau sind und wo ihre Grenzen zu 
anderen Handlungen liegen (Hacking 1991). Es gibt verschiedene Definitionen des Phänomens, wie z.B. das eng gefasste ,battered child syndrome“, bei dem der Fokus auf diagnostizierbaren (schweren) Verletzungen liegt, bis hin zu weiten Auslegungen, die auf alle gewaltförmigen elterlichen Erziehungshandlungen abzielen, unabhängig davon, ob diese feststellbare Beeinträchtigungen zur Folge haben (Engfer 1986, S. 2). Bei Eberl et al. (2010, S. 139) heißt es z. B. in Anlehnung an TrubeBecker: „Kindesmisshandlung ist die gewaltsame, nicht unfallbedingte, körperliche oder seelische Schädigung eines Kindes durch aktives Handeln oder durch Unterlassen einer erwachsenen Beziehungs- oder Betreuungsperson. Als Folgen für das Kind können Verletzungen, Fehlentwicklungen oder Tod eintreten“.

Es zeigt sich, dass im medizinischen Kinderschutz inzwischen eher weite Definitionen von Gewalt und Misshandlung bevorzugt werden. Bei Frank (1989, S. 16) heißt es z.B.: „Für Ärzte empfiehlt es sich, von einer weitgefassten Begriffsbestimmung bei der Einschätzung von Fällen auszugehen, um einer möglichst großen Zahl von gefährdeten Kindern Hilfe zuteil werden zu lassen“. Dies macht verständlich, dass die Arbeit dieser Kinderschutzgruppen nicht so sehr durch Fälle von schweren Misshandlungen geprägt ist, sondern vor allem durch ,uneindeutige Fälle, die weiterer Abklärung bedürfen und weniger schwere Fälle, in denen die Erziehungskompetenz der Eltern eine zentrale Rolle spielt" (Inthorn 2012, S. 75). Trotz der Bevorzugung eines breiten Verständnisses von Misshandlung - oder vielleicht auch gerade deswegen -, scheint es innerhalb der Medizin große Varianzen in der Wahrnehmung darüber zu geben, was Misshandlung konkret ist. In Bezug zu empirischen Studien über das sehr unterschiedliche Vorkommen von entsprechenden Fällen in Arztpraxen heißt es bei Hermann et al. (2016, S. 362), dass daran deutlich werde, ,wie unscharf die Definition von Kindesmisshandlung und Vernachlässigung oder Kindeswohl ist und dass das Vorgehen wenig standardisiert und uneinheitlich ist".

Auch wenn es in der Medizin eine Tendenz dazu gibt, Kindesmisshandlung als ein rein medizinisches Phänomen zu beschrieben, hat das Konzept zudem einen kulturellen Aspekt. ${ }^{2}$ Dieser zeigt sich besonders in der Unterscheidung zwischen „legitimen Erziehungsmaßnahmen“ und „Misshandlungen“, welche „stark von gesellschaftlichen Normen und anerkannten Erziehungspraktiken beeinflusst" ist und sich z. B. darin zeigt, ,dass Anfang des 20. Jahrhunderts solche Erziehungsmethoden anerkannt waren, die heute uneingeschränkt unter den Tatbestand Kindesmisshandlung fallen würden“ (Mertens und Pankofer 2011, S. 27). ${ }^{3}$ Kurz und prägnant hat es der britische Pädiater Meadow (1989, S. 727) beschrieben: „A child is considered to be abused if he or she is treated by an adult in a way that is unacceptable in a given culture at a given time“.

Eine weitere bedeutsame Unterscheidung ist die zwischen Unfällen und Misshandlungen. Begrifflich und differenzialdiagnostisch werden misshandlungsbeding-

\footnotetext{
${ }^{2}$ Im Gegensatz zu der Anfangszeit des medizinischen Kinderschutzes wird Kindesmisshandlung heutzutage in der Regel nicht mehr so strikt definiert. Bei Hermann et al. (2016, S. 2) heißt es z. B.: ,Kindesmisshandlung ist keine eigene Erkrankung im engeren Sinne.“.

3 Insbesondere Pfohl (1977) und Hacking (1991) weisen darauf hin, dass es u.a. ein Anliegen des medizinischen Kinderschutzes ist, normativ auf diese Unterscheidung einzuwirken. Hacking (1991, S. 287) charakterisiert deshalb child abuse als „framework of normalcy and pathology“.
} 
te Verletzungen von Verletzungen abgegrenzt, die durch Unfälle entstanden sind sowie von Befundkonstellationen, die durch Krankheiten verursacht werden. Aber die Verletzungen, welche zu einer Missbrauchsdiagnose führen können, sind oftmals mehrdeutig und können sowohl auf einen Unfall als auch auf eine Misshandlung hindeuten. Aus medizinischer Sicht ist deshalb ,[d]ie Diskrepanz zwischen angegebenem Vorfall und dem vorliegenden klinischen Verletzungsbefund" (Hermann et al. 2016, S. 24) ein Kardinalshinweis für eine Misshandlung. Auslöser für den Verdacht auf eine Misshandlung sind also oftmals nicht die Verletzungen selbst, sondern unplausible Verletzungserklärungen. Ganz ähnlich beschreibt Franzheld (2017) das Aufkommen eines ärztlichen Verdachts auf eine Kindeswohlgefährdung. Dieser entstehe erst durch ,die Sinnverknüpfung körperbasierter Verletzungsspuren mit gesprächsbasierten Verletzungsgeschichten, die im ärztlichen Anamneseprozess thematisch werden“ (Franzheld 2017, S. 150). Eine Grundlage dafür ist eine gewisse Distanzierung von den Eltern und ihren Erklärungen zum Verletzungshergang. Bereits Pfohl (1977, S. 319) hat darauf hingewiesen, dass sich durch die Übernahme von kinderschutzbezogenen Aufgaben in der Medizin die Stellung der Eltern gegenüber den Ärzt*innen verändert und beschrieb diese Verschiebung wie folgt: „Problems associated with perceiving parents as patients whose confidentiality must be protected where reconstructed by typifying them as patients who need help." Dem ließe sich im Hinblick auf die Abgrenzung von Unfällen im medizinischen Konzept von Misshandlung hinzufügen, dass dadurch Patien*tinnen potenziell nicht nur als Personen gerahmt werden, welche aufgrund von Überforderung Hilfe benötigen, sondern auch als Personen, welchen - zumindest zeitweise - misstraut werden muss, um eine mögliche Misshandlung aufzudecken.

\subsection{Zur Diagnose von Misshandlung und Gewalt}

Das Identifizieren einer Misshandlung ist eine komplexe Aufgabe. Wie Söhner et al. (2017, S. 74) herausstellen, ist die Aufgabe des medizinischen Kinderschutzes eine Doppelte: Auf der einen Seite gilt es, mögliche Indizien für eine Misshandlung durch eine fachgerechte Diagnose festzustellen. Auf der anderen Seite soll die Diagnose eine Prognose für weitere mögliche Gefährdungen leisten, um das Kind in diesem Fall vor ihnen zu schützen. Für die Diagnostik - und im Kinderschutz wegen seiner weitreichenden Konsequenzen nochmal verschärft - gilt außerdem, dass durch sie „ein Krankheitsbild intersubjektiv nachvollziehbar und gleichzeitig für andere Untersuchende reproduzierbar“" (Söhner et al. 2017, S. 74) gemacht werden soll. Wie Söhner et al. (2017, S. 76) weiter herausstellen, sind die medizinischen Krankheitsbilder keinesfalls statische Gebilde, sondern unterliegen einem Wandel, der sich auf die entsprechenden Suchstrategien zur Aufspürung dieser Krankheitsbilder auswirkt - also auf die Art und Weise ihrer Diagnostik und der praktischen Herstellung von Evidenz von Krankheits- oder Misshandlungsbefunden.

Schon die fachgerechte Befundstellung bei einem Misshandlungsverdacht scheint der medizinischen Praxis eine doppelte Perspektive zu verleihen, welche mit besonderen Voraussetzungen für die Diagnostik einhergehen. Verletzungen, wie Verbrühungen und Knochenbrüche, können unabhängig von ihrer Ursache (Misshandlung oder Unfall) behandelt werden. Typisch für eine Misshandlungsdiagnostik ist also 
nicht nur die fachgerechte Bestimmung der vorliegenden Verletzungen, um diese zu behandeln, sondern auch der Versuch, die eigentliche Ursache der Verletzung aufzuklären. Diesbezüglich ließe sich eine Misshandlungsdiagnose als eine „Doppeldiagnose“ oder „Hybriddiagnose“ charakterisieren. Denn zum einen müssen Verletzungen erkannt und behandelt und zum anderen darüber hinaus die Möglichkeit einer Misshandlung als Ursache der Verletzungen in Betracht gezogen und differenzialdiagnostisch geprüft werden. Notwendig wird damit eine Wissensbasis, die über die Bestimmung und Behandlung von „normalen“ Verletzungen hinausgeht. Damit ist dem Konzept immer die Möglichkeit eingeschrieben, dass ein Verdacht nicht aufkommt und Anzeichen für Misshandlungen übersehen werden. Dies wird in der Medizin als falsch-negative Diagnose bezeichnet. Ein weit gefasster Begriff von Misshandlung erschwert in diesem Zusammenhang, alle diagnostischen Kriterien zu kennen und eine Differenzialdiagnose durchzuführen. Mit einem weiten Begriff erhöht sich zudem das Risiko von falsch-positiven Fällen, wenn z.B. Verletzungen, die von einem Unfall verursacht wurden, fälschlicherweise von Ärzt*innen als misshandlungsbedingte Verletzungen interpretiert werden (Engfer 1986, S. 1 ff.).

Mit der Vergrößerung der notwendigen Wissensbasis scheint die Fehleranfälligkeit zu steigen. Franzheld (2017, S. 152ff.) schreibt in seiner Untersuchung des medizinischen Kinderschutzes, dass die Integration von Praktiken der Gewalterkennung dem medizinischen Handeln eine zweite Suchbewegung hinzufügt, welche auf die Ermittlung der Ursache der Verletzung gerichtet ist. Franzheld (2017, S. 152ff.) zufolge entstehen zwei unterschiedliche Rahmensetzungen während der Verdachtsbildung - Verletzung und Gefährdung -, zwischen denen die Ärzt*innen beständig wechseln, um den Fall zu beurteilen. Die Betrachtung des Falles im Gefährdungsrahmen - also als möglicher Fall von Kindeswohlgefährdung - führt ,nicht nur zu einer erheblichen Komplexitätssteigerung der Beobachtung, sondern überhaupt erst zu einer verdachtsbezogenen Perspektivenöffnung“ (Franzheld 2017, S. 161).

Während die erste Suchbewegung auf die Erkennung und Behandlung der Verletzungen des Kindes aus ist, schließt die zweite Suchbewegung die Sorgeberechtigten mit ein. Da misshandlungsbedingte Verletzungen oftmals nicht als solche vorgestellt werden, sondern versucht wird, diese als Folge eines Unfalls auszugeben, muss das medizinische Personal versuchen, diese „Deckbehauptungen“ der Sorgeberechtigten zu dechiffrieren. ${ }^{4}$ Um Spuren von Gewalt gegen Kinder im medizinischen Kontext zu erkennen, erhalten die Ärzt*innen eine Art Ermittlungsfunktion. Bereits Kempe et al. (1962, S. 19) haben in ihrem initialen Aufsatz herausgestellt, dass die Rolle der Ärzt*in bei der Beurteilung eines Verdachtes auf Misshandlung der einer Polizist*in ähnelt, die versucht, ein Verbrechen aufzuklären. Nach Kempe et al. (1962, S. 19) ist diese Ermittlungsfunktion nicht einfach vereinbar mit der weiteren medizinischen Ausbildung und Praxis. Denn das Einnehmen dieser „Ermittlungshaltung“ verändert das „traditionell ausschließlich auf ,Krankheit“ bezogene Berufsprofil“" (Bode und Turba 2014, S. 207) der Ärzt*innen, in dem eher die Behandlung von Symptomen als von Ursachen (Conrad und Schneider 1980, S. 163) im Vordergrund steht. Nach

\footnotetext{
4 Diesbezüglich heißt es in einem Text des Kindermediziners von Bismarck (2013, S. 30 f.): ,Misshandelte Kinder werden zwar häufig wegen der dabei erlittenen Verletzungen ärztlich vorgestellt, dabei wird der eigentliche Vorfall aber meistens mit einer erfundenen Unfallgeschichte getarnt“.
} 
Bode und Turba (2014, S. 207) wird das von den Ärzt*innen als Hybridisierung ihrer Berufsrolle wahrgenommen, welche in Bezug auf das Vertrauensverhältnis zu ihren Patient*innen problematisch sein kann.

Relativ unabhängig davon, ob für verschiedene Formen von Misshandlungen eher enge oder weite Definitionen verwendet werden, gibt es bei ihrer Ermittlung bzw. Diagnose zum Teil sehr unterschiedliche Probleme. Unabhängig von der genauen Definition ist ein sexueller Missbrauch im Vergleich zu einer physischen Misshandlung ein schwerer (medizinisch) fassbarer Gegenstand. Debertin und Banaschak (2013, S. 5) stellen bei der Definition des sexuellen Missbrauchs von Kindern heraus, dass der Begriff „,bezüglich seines Inhaltes unscharf“ ist und von sog. „Handson“- bis zu „Hands-off“-Handlungen ,ein sehr breites Spektrum an Handlungen“ umfasst. Dies bedeutet, dass es in diesem Zusammenhang ,keinen einheitlichen körperlichen Untersuchungsbefund bei Kindern geben wird" (Debertin und Banaschak 2013, S. 5). Bei einer physischen Misshandlung scheint es sich um ein begrifflich und praktisch-diagnostisch fassbareres Phänomen zu handeln als bei einem sexuellen Missbrauch (vgl. Jud et al. 2009). Auch Inthorn (2012, S. 70) stellt fest, dass „sich der Verdacht auf körperliche Gewalt durch den Abgleich der Verletzungsmuster mit dem Bericht des Unfallhergangs insbesondere bei Säuglingen und Kleinkindern deutlich einfacher erhärten oder entkräften [lässt] als ein Verdacht auf sexuellen Missbrauch“.

Eng verbunden mit dieser praktisch-diagnostischen Fassbarkeit der verschiedenen Misshandlungsformen ist die Möglichkeit zur Herstellung von Evidenz aufgrund der Untersuchungsbefunde. Inthorn (2012, S. 70) weist darauf hin, dass bei einem Verdacht auf einen sexuellen Missbrauch ,in der Regel nur Indizien vorhanden [sind], die keine eindeutigen Schlüsse, weder in Richtung einer Bestätigung noch einer endgültigen Entkräftung des Verdachts, zulassen“. Während für den Verdacht auf eine physische Misshandlung durch eine medizinische Untersuchung prinzipiell eine Verifikation und eine Falsifikation möglich sind, ist für einen Verdacht auf sexuellen Missbrauch i.d.R. nur eine Verifikation möglich. Dies zeigt ein Blick auf verschiedene Untersuchungen zu den Aufklärungsquoten von Verdachtsfällen in medizinischen Einrichtungen (Thun-Hohenstein 2006; Jud et al. 2009; Eberl et al. 2010; Inthorn 2012). Diese geben Hinweise auf die Grenzen einer medizinischen Diagnostik und zeigen, dass ein Verdacht auf Misshandlung oftmals nicht abschließend geklärt werden kann, also weder falsifizierbar oder noch verifizierbar ist. Die Zahlen schwanken zwischen 30 bis ca. $60 \%$ der Fälle, in denen ein Verdacht nicht geklärt werden kann, und daher auch zwischen ca. 25 und $60 \%$ der Fälle, in denen ein Verdacht als bestätigt angesehen wird (vgl. Eberl et al. 2010; Inthorn 2012). In den Studien von Thun-Hohenstein (2006) und Jud et al. (2009) werden außerdem die Aufklärungsquoten für verschiedene Verdachtsarten weiter aufgeschlüsselt. Dabei wird ersichtlich, dass einer medizinischen Diagnostik bei Verdachtsfällen auf einen sexuellen Missbrauch engere Grenzen gesetzt sind. Ein Verdacht auf sexuellen Missbrauch kann nach den Statistiken seltener falsifiziert und verifiziert werden als ein Verdacht auf physische Misshandlung. Die Studie von Thun-Hohenstein (2006) weist ergänzend darauf hin, dass ein relevanter Unterschied zwischen beiden Verdachtsarten in der Auffindbarkeit klinisch relevanter Diagnosen liegt. Bei einem 
Verdacht auf sexuelle Misshandlung wurden diese, im Vergleich zu einem Verdacht auf physische Misshandlung, nur halb so oft festgestellt.

Beide Formen von Gewalt unterscheiden sich zudem darin, wie ein Verdacht aufkommt. Bei Jud et al. (2009) wird angemerkt, dass ein Verdacht auf physische Misshandlung eher durch das Krankenhauspersonal aufkam, wobei ein Verdacht auf sexuellen Missbrauch der Kinderschutzgruppe eher von außerhalb gemeldet wurde. Auch Eberl et al. (2010, S. 141) weißen darauf hin, dass ein „Großteil (88\%) [der Fälle bei denen ein sexueller Missbrauch angenommen wird] [...] durch sekundäre Somatisierung, Suizidversuch oder im Rahmen von Routineuntersuchungen aufgedeckt“" wurde. Während es demnach spezifische Symptome (wie z.B. geformte Hämatome) und oftmals damit einhergehende unplausible Verletzungserklärungen gibt (wie z.B. das Kind sei von der Couch gefallen, während niemand zugegen war), welche auf eine physische Misshandlung hindeuten können und prinzipiell die Überprüfung des Verdachts erlauben, ist dies bei einem sexuellen Missbrauch wesentlich seltener der Fall. Nach Hermann et al. (2016, S. 136) sollen bei Kindern, welche Opfer von sexueller Gewalt wurden, in 90-95\% der Fälle körperliche Normalbefunde, also keine besonderen körperlichen Verletzungen, gefunden worden sein. Bei einem Verdacht auf einen sexuellen Missbrauch dient eine medizinische Untersuchung demzufolge eher dazu, die Aussage des Kindes zu validieren, welche weit häufiger zu der Diagnose eines Missbrauchs führt als die medizinische Untersuchung selbst. Hingegen können die Ergebnisse der medizinischen Untersuchung bei einem Verdacht auf eine physische Misshandlung zum einen ohne eine Verletzungsnarration eine Aussagekraft besitzen und zum anderen dazu verwendet werden, die Verletzungserklärung der Sorgeberechtigten zu verifizieren oder zu falsifizieren. ${ }^{5}$

Die Grenzen einer medizinischen Diagnostik zeigen sich ebenfalls bei der Frage, ob ein Verdachtsfall dem Jugendamt oder der Justiz gemeldet werden soll. Besonders bei diesem Problem soll die Beobachtung und Bewertung der Eltern-KindInteraktionen, also eine Sozialanamnese, der zentrale Beurteilungsmaßstab sein, da hierfür die reinen medizinischen Befunde als nicht aussagekräftig genug angesehen werden (Inthorn 2012, S. 57 ff..). Inthorn (2012, S. 59) zufolge wird gerade bei Fällen mit interkulturellen Unterschieden ,deutlich, dass die Kinderschutzgruppen bei der Einschätzung der Eltern auf Alltagswissen und allgemeine gesellschaftliche Vorstellungen von Normalität oder verantwortlicher Elternschaft zurückgreifen müssen, die sich aus ihren persönlichen Erfahrungen speist. Kinder aus Familienstrukturen, die der Kinderschutzgruppe vertrauter sind (kein Migrationshintergrund, keine Unterschichtenproblematik) können daher differenzierter betrachtet werden“.

\footnotetext{
5 Dementsprechend wird z. B. im Handbuch von Hermann et al. (2016) die Aussage des Kindes hervorgehoben, wenn es um die Diagnostik bei dem Verdacht eines sexuellen Missbrauchs geht. Im Kapitel zur Diagnostik bei einem Verdacht auf körperlicher Misshandlung wurde die Aussage des Kindes hingegen nicht erwähnt, sondern nur die Erklärung der Eltern über die Entstehung der Verletzung.
} 


\section{Zur Rolle der medizinischen Forschung}

In Bezug auf die Herstellung von Evidenz ist noch eine weitere Entwicklung relevant. Nach Hermann et al. (2016, S. 24) ist die elementare Voraussetzung für die Diagnose von Misshandlungen aufgrund medizinischer Befunde ,[d]ie fachgerechte und wissenschaftlich abgesicherte Befunderhebung, Interpretation, Diagnose und Differenzialdiagnose“. Dabei ist die fachgerechte und wissenschaftliche Befunderhebung einem steten Wandel unterworfen. Dies wird z.B. im Zusammenhang mit der Bewertung von Hämatomen für die Diagnose einer physischen Misshandlung deutlich. Dazu heißt es bei Hermann et al. (2016, S. 66): „Für die zeitlich exakte und forensisch zuverlässige Zuordnung des Hämatomalters aufgrund seiner Farbe gibt es nach neueren Daten keine wissenschaftliche Evidenz. Eine pseudogenaue Altersfestlegung sollte in Misshandlungsfällen strikt vermieden werden“. Hier wird deutlich, dass vorher wohl übliche Praktiken durch weitere Forschung zu ,,pseudogenauen" Praktiken werden können. Der Versuch der Altersfestlegung von mehreren Hämatomen zielte darauf, durch den Nachweis eines unterschiedlichen Alters der Hämatome das wiederholte Zufügen von Verletzungen zu beweisen. Dieses Unterfangen wurde durch die neuere wissenschaftliche Evidenz folglich limitiert. Auf der anderen Seite vereinfacht es die Dokumentation der medizinischen Befunde, da eine möglichst genaue Dokumentation der Farbe der Hämatome dadurch weniger relevant wird - als bedeutender wird die genaue Beschreibung der Position, Form und Größe der Hämatome angesehen.

Wie wissenschaftliches Wissen im Allgemeinen sind „die nosologischen Klassifikationssysteme selbst nicht statisch, sondern ständigen Wandel unterworfen" (Söhner et al. 2017, S. 76). Wie Söhner et al. (2017, S. 76) an dieser Stelle ausführen, verändert sich ,,[m]it solchen Verschiebungen innerhalb der nosologischen Klassifikation [...] auch der diagnostische Zugang, d.h. die Suche nach systematisch erfassbaren Zeichen“. In ihrer Untersuchung des medizinischen Wissens zum Thema Kindesmisshandlung machen die Autoren diesbezüglich verschiedene Tendenzen aus: Zum einen die Transformation von Zeichen mit einer relativ klaren und eindeutigen Beweiskraft für die Diagnose von Misshandlungen zu Zeichen, welche nur noch eine relative Beweiskraft besitzen. Charakteristisch an der frühen Forschung zu dem Thema war das Anliegen, einzelne Anzeichen zu identifizieren, die exklusiv für eine bestimmte Missbrauchsdiagnose sind. Im medizinischen Sprachgebrauch werden diese Zeichen als pathognomonische Zeichen bezeichnet. Spätere Forschungen haben jedoch ergeben, dass die als pathognomonisch angenommenen Zeichen mehrdeutig sein können. Sie haben dadurch ihren exklusiven Charakter größtenteils eingebüßt. Damit hat sich die fachgerechte Befunderhebung verändert. Es ist nicht mehr ,state of the art“, auf der Grundlage von einzelnen Zeichen eine Diagnose zu stellen, sondern dies anhand eines spezifischen Sets an Zeichen durchzuführen, welche erst in ihrer Kombination einen hohen diagnostischen Wert erhalten. Söhner et al. charakterisieren diese Tendenz auch als eine Ausdifferenzierung der Beweiskraft von Zeichen im medizinischen Wissen um Kindesmisshandlung.

Illustrieren lässt sich diese Ausdifferenzierung der Beweiskraft von einzelnen Zeichen bspw. an der Verwendung des Begriffs ,pathognomonisch“ in dem deutschen Handbuch zum Thema Kindesmisshandlung von Herrmann et al. (2016). Das 
Wort wird dort insgesamt sieben Mal verwendet. Dabei wird es aber nicht seinem eigentlichen Sinn nach gebraucht - als eindeutiger Hinweis eines Zeichens für einen Befund -, sondern um darauf hinzuweisen, dass ein bestimmtes Zeichen aufgrund neuerer Forschung inzwischen nicht mehr als pathognomonisch für einen besonderen Befund anzusehen ist (Herrmann et al. 2016, S. 51, 56, 108). Auf der anderen Seite wird darauf hingewiesen, dass ein bestimmtes Zeichen für sich noch kein pathognomonischer Hinweis auf einen Missbrauchsbefund ist, sondern eher ein (starker) Hinweis darauf - vor allem, wenn es keine plausible Verletzungserklärung gibt (Herrmann et al. 2016, S. 43, 58, 96, 113). Der Begriff pathognomonisch wird in diesem Handbuch in Anführungsstrichen verwendet, um besonders darauf hinzuweisen, dass einem Zeichen, welches früher als pathognomonisch galt, dieser Status nicht mehr zukommt.

Ein weiteres instruktives Beispiel ist das sog. „Adams-Schema“. Es ist ein Klassifikationssystem zur Interpretationshilfe von Befunden in Bezug auf ihre Aussagekraft zur Diagnosestellung eines möglichen sexuellen Missbrauchs, welches seit seiner ersten Veröffentlichung 1992 regelmäßig überarbeitet und modifiziert wird, um dem jeweiligen Stand der Forschung Rechnung zu tragen (Hermann et al. 2016, S. 164f.). Nach Hermann et al. (2016, S. 164) hat es sich ,zu einem populären und vielgenutzten Schema für die Beurteilung von klinischen wie auch Laborbefunden entwickelt“. Söhner et al. (2017, S. 83f.) zeigen in einer Analyse der verschiedenen Versionen des Schemas, dass im Laufe der Zeit die Kategorie Clear Evidence of Penetrating Injury, die es bis 1994 gab, in späteren Versionen nicht mehr vorkommt. Die höchste Kategorie ist seit 2002 Findings diagnostic of trauma and/or sexual contact. Söhner et al. (2017, S. 84) schlussfolgern, dass „,[d]ie Möglichkeit, einen hundertprozentigen Beweis durch ein einzelnes Indiz zu erbringen, [...] nun deutlich eingeschränkter" ist.

Neben der Tendenz zur Ausdifferenzierung der Beweiskraft von Zeichen gibt es auf der anderen Seite Zeichen, die im Verlauf der Zeit relativ konstant geblieben sind. Das gilt größtenteils für die radiologischen Befunde, wie z. B. der Nachweis von multiplen Knochenbrüchen, die über die Zeit hinweg kaum an Beweiskraft eingebüßt haben. Interessanterweise haben sich gerade die Zeichen am beständigsten in ihrem Geltungsbereich gezeigt, ,die schon vor der radiologischen Ära in der Diagnostik von Misshandlungen als aussagekräftige Belege gewertet wurden“ (Söhner et al. 2017, S. 83). Dazu gehören insbesondere geformte Hämatome, welche durch ihre besondere Form auf den Verletzungsgegenstand schließen lassen.

Interessant ist ein Vergleich dieser Dynamiken mit den Entwicklungen der Definitionen von verschiedenen Formen von Misshandlungen. Dadurch drängt sich die These auf, dass die Tendenz zur Ausdifferenzierung der Aussagekraft diagnostischer Zeichen vor allem den Bereich des sexuellen Missbrauchs betrifft, welcher dem Konzept der Kindesmisshandlung erst später hinzugefügt wurde. Auf der anderen Seite haben sich gerade die diagnostischen Zeichen, die auf eine körperliche Misshandlung hinweisen und zum ,ursprünglichen“ Konzept von Misshandlung gehören, als relativ stabil in ihrer Beweiskraft gezeigt. Nach Görgen et al. (2013, S. 223) waren es gerade die damals neuen radiologischen Befunde, welche den ,Medizinern die empirische Sicherheit [verschafften], verschiedene Verletzungen unterschiedlichen Alters miteinander und mit Misshandlungen in Verbindung zu bringen“. Und es 
scheint so, als ob diese Befunde weiterhin diese empirische Sicherheit zur Diagnose von physischem Missbrauch besitzen. Sie wurden durch nachfolgende Forschungen offenbar eher bestätigt als widerlegt. Eine andere Dynamik zeigt sich in der Forschung zum sexuellen Missbrauch. Nach Hermann et al. (2016, S. 158) gibt es auf der einen Seite ,eine deutliche Zunahme der Wertung von Befunden als normal oder unspezifisch“ und auf der anderen Seite „hat die Zahl der Befunde, die eindeutig einem penetrierenden Trauma zugeordnet werden, stark abgenommen".

\section{Zusammenfassung und Ausblick}

Ziel des Beitrages ist es einerseits einen Überblick über medizinische Definitionen von Misshandlungen und Praktiken der Gewalterkennung zu geben sowie andererseits aufzuzeigen, dass diese Definitionen und Praktiken bestimmte Wandlungsprozesse durchlaufen haben. Wie Burri und Dumit (2007, S. 3) für die Biomedizin beschreiben, lässt sich der medizinische Kinderschutz als ein Beispiel für eine Verwissenschaftlichung der Medizin ansehen. Der medizinische Kinderschutz ist von Anfang an eng mit der medizinischen Forschung und medizinisch-technischen Innovationen, wie dem Röntgenapparat und dem Kolposkop, verwoben. Gleichzeitig gibt es in dem medizinischen Feld starke Bemühungen, die Praxis auf eine evidenzbasierte Grundlage zu stellen. Weiterhin gilt für diese mit dem medizinischen Kinderschutz verbundenen Dynamiken: ,these processes reconfigure the epistemic tools, the material procedures, and the social relations and infrastructures in biomedicine and exert a major impact on society in general" (Burri und Dumit 2007, S. 3$){ }^{6}$

Der Kinderschutz wurde als ein medikalisiertes Feld beschrieben, in dem die Medizin eine besondere Deutungshoheit innehat, insbesondere bei der Frage, was Gewalt ist und wie Auswirkungen von Gewalt aufgedeckt werden können. In einem historischen Überblick habe ich versucht herauszustellen, dass sich die Beschäftigung der Medizin mit dem Thema der Gewalt gegen Kinder und die Etablierung einer Deutungshoheit auf diesem Gebiet aus einem sehr spezifischen Kontext heraus entwickelt hat. Verschiedene Faktoren, wie z. B. die Weiterentwicklungen in der Radiologie, die Möglichkeit zur Bündelung von Fällen im Krankenhaus und die Auffassung, dass es sich bei Kindesmisshandlung um einen genuin medizinischen Gegenstand handelt, spielen hierfür eine entscheidende Rolle.

Zentral für die Argumentation ist die Annahme, dass die Kinderschutzmedizin verschiedene Wandlungsprozesse durchlaufen hat, die mit Steigerungen von Komplexität gekoppelt sind. Mit der Erweiterung der Definition von Misshandlung von weitestgehend intentionaler, physischer Gewalt um Vernachlässigung und sexuellen Missbrauch kam es nicht nur zu einer „Entgrenzung der medizinischen Einflusssphäre" (Görgen et al. 2013, S. 223f.) und zu einer Ausweitung des Kreises der potenziellen Opfer von Gewalt und Misshandlungen (Hacking 1991, S. 272). Es vergrößerte sich ebenfalls die notwendige Wissensbasis, um mögliche Spuren von Gewalt überhaupt wahrnehmen und nachweisen zu können, womit gleichzeitig die

\footnotetext{
${ }^{6}$ Zu gesellschaftlichen Auswirkungen siehe z. B. Hacking (1991, 1999).
} 
Fehleranfälligkeit der Diagnosen angestiegen ist (vgl. Engfer 1986). Zudem führte die medizinische Forschung zu einer Steigerung von Komplexität, welche das Wissen um die Charakteristika und Möglichkeiten der Diagnose von unfalltypischen und misshandlungsbedingten Verletzungen ausdifferenziert hat. Dies führt da$\mathrm{zu}$, dass als pathognomonisch angenommene Zeichen für Misshandlungsdiagnosen oftmals ihren exklusiven diagnostischen Stellenwert verloren haben. Dadurch ist für eine fachgerechte Begründung einer Misshandlungsdiagnose nicht mehr die Ermittlung einzelner, spezifischer, sondern eines spezifischen Sets an Zeichen notwendig, welche erst durch ihre Kombination einen hohen diagnostischen Wert bekommen (Söhner et al. 2017).

Eine Komplexitätssteigerung durch den Einbezug von Praktiken der Gewalterkennung wurde für den medizinischen Blick ausgemacht, was hier als Prozess der Hybridisierung charakterisiert wird (Bode und Turba 2014; Franzheld 2017). Der medizinische Blick ist darauf gerichtet, die eigentlichen Ursachen der vorgefundenen Verletzungen zu ermitteln (Conrad und Schneider 1980). Verbunden ist damit eine Transformation der Stellung der Sorgeberechtigten gegenüber den Ärtzt*innen von ,patients whose confidentiality must be protected“ zu ,patients who need help“ (Pfohl 1977, S. 319). Während sich bei frühen Konzeptionen von child abuse ein Verdacht auf die Eltern oder Sorgeberechtigten als Verursacher*innen von Verletzung durch die Ausübung von Gewalt gegen ihre Kinder richtete, verschiebt sich dieser Verdacht teilweise durch die Erweiterung des Konzepts auf die Erziehungsfähigkeit der Eltern (Intorn 2012). Relativ konstant scheint dabei die Ansicht zu bleiben, dass nicht nur die von child abuse betroffenen Kinder professionelle Hilfe benötigen, sondern potenziell ebenfalls ihre Eltern oder Sorgeberechtigten (vgl. Pfohl 1977; Hacking 1991).

Das durch den Einbezug von Praktiken der Gewalterkennung neu Hinzukommende - der Prozess der Hybridisierung - lässt sich anlehnend an Kempe et al. (1962, S. 19) als eine Art polizeilicher Ermittlungs- und Kontrolllogik bezeichnen. Vor diesem Hintergrund sind z.B. die Ergebnisse der Studie von Turba (2018) zur Polizei im Kinderschutz interessant. Relativ analog zu den hier vorgestellten Ergebnissen stellt er fest, dass mit der Einbindung von Kinderschutz bezogenen Aufgaben in die Arbeit der Polizei eine Steigerung der Komplexität der Arbeit einhergeht (Turba 2018, S. 361). Die Polizist*innen erhalten dadurch eine hybride Position, welche durch den Einzug von quasi-pädagogischen Tätigkeiten in ihr Tätigkeitsspektrum gekennzeichnet ist (Turba 2018, S. 362). Diese Hybridität steht wiederum z. T. im Konflikt mit dem Bild der/des ,richtigen“ Polizist*in. Turba stellt weiterhin heraus, dass das nicht heißt, dass Polizist*innen dadurch zu Sozialarbeiter*innen werden, die Entgrenzung also spezifische Grenzen hat. Dies ist auch für den medizinischen Kinderschutz anzunehmen. Durch ihn werden Ärzt*innen nicht gleich zu Polizist*innen. Der Prozess lässt sich als ein Eindringen eines staatlichen Kontrollauftrags in die Medizin interpretieren. Außerdem hat die hier beschriebene Hybridisierung der Medizin spezifische Grenzen, welche bereits ein Hemmnis bei der ursprünglichen Formation des medizinischen Kinderschutzes gewesen sind, insbesondere das Problem des Vertrauensverhältnisses zu den eigenen Patient*innen. Vor diesem Hintergrund ist es kein Zufall, dass der medizinische Kinderschutz sozusagen an seinem Ge- 
burtsort, dem Krankenhaus, weitaus stärker verbreitet ist als bei niedergelassenen Ärzt*innen, die oftmals eine nähere Beziehung zu den Patient*innen pflegen.

Anschlussfähig ist das hier Aufgezeigte weiterhin an organisationssoziologische Perspektiven. Die Integration von Praktiken der Gewalterkennung hat Auswirkungen auf die Arbeitsorganisation im Krankenhaus. Verbunden mit der Komplexitätssteigerung in der Beobachtung der Fälle (Franzheld 2017, S. 161) ist eine Unsicherheit bei der Bewertung der Fälle. Die Etablierung des Kinderschutzes im Krankenhaus ließe sich wahrscheinlich als eine organisationsintern erzeugte Unsicherheit interpretieren, selbst wenn Ansprüche aus der Organisationsumwelt sicher eine (immer größere) Rolle spielen (Apelt und Senge 2015). ${ }^{7}$ Vor diesem Hintergrund erscheinen die Ausbreitung von Kinderschutzgruppen in Krankenhäusern als ein Mittel zur Reduktion von Unsicherheit, die durch Aufgaben des Kinderschutzes entstandenen sind. Durch diese Gruppen soll die Bearbeitung von Verdachtsfällen im Krankenhaus weiter institutionalisiert werden, indem die Entscheidungen nicht mehr von Einzelpersonen, sondern von Gruppen (zu denen oftmals Pfleger*innen und Sozialarbeiter*innen gehören) getragen werden, um Potenziale zur Gewalterkennung in diesen Organisationen besser auszuschöpfen (Intorn 2012, S. 43 f.).

Anschließen lässt sich weiterhin an Beobachtungen und Analysen zu Veränderungsprozessen im Krankenhauswesen (Bode und Vogd 2016). Vor dem Hintergrund einer Diagnose von „Dauerstress“ in vielen Häusern aufgrund eines gestiegenen ökonomischen Drucks scheint die Durchsetzung des medizinischen Kinderschutzes im Krankenhaus quer zu dieser Dynamik zu liegen. Denn medizinischer Kinderschutz kann bisher kaum kostendeckend betrieben werden. Trotz bestimmter Fortschritte in der Finanzierung der medizinischen Kinderschutzarbeit (Schwier et al. 2019) ist „,der entstehende Aufwand [...] meist nicht durch die für die normale Gesundheitsversorgung zuständigen Kostenträger (Krankenkassen) gedeckt" (Bode und Turba 2014, S. 83), weshalb diese Aufgaben oftmals als „Schattenleistungen“ erbracht werden und von impliziten Querfinanzierungen abhängig sind. Auch die zeitlichen Ressourcen des (medizinischen) Personals werden durch ihn weiter verknappt. Es handelt sich um eine oftmals emotional anspruchsvolle Arbeit, die häufig zusätzlich zur ,eigentlichen“ medizinischen Tätigkeit geleistet wird und oft nicht Bestandteil des Stellenprofils ist. Kurzum trägt der medizinische Kinderschutz sogar noch zu einer Verschärfung des Dauerstresses in Krankenhäusern bei und der ökonomische Druck wird durch ihn eher größer als kleiner. Die fortschreitende Etablierung der Kinderschutzmedizin lässt sich, obwohl sie einer ökonomischen Logik zuwiderläuft, deshalb als Hinweis dafür ansehen, dass es trotz der soziologischen Überlastungsdiagnose des Krankenhauswesens dort weiterhin Resilienzpotenziale gibt und Veränderungsprozesse vonseiten der Ärzt*innenschaft (z. T. mit der Unterstützung durch Pflege, Soziale Arbeit) nach eigenen, medizinischen Kriterien in Gang gebracht werden. Es lässt sich also fragen, ob den Ärzt*innen nicht vielleicht entgegen z. B.

\footnotetext{
7 Nach Bode und Turba (2014, S. 84f.) gibt es zwar gewisse Kooperationserwartungen an das Gesundheitswesen in deutschen Kinderschutzsystem, aber in Hinsicht auf institutionelle Bestimmungen keine formale Zuständigkeit für den Kinderschutz. Eine Ausnahme ist $\$ 4$ des KKG (Gesetz zur Kooperation und Information im Kinderschutz) in dem auch Ärzt*innen u. a. aufgefordert, aber nicht verpflichtet werden, möglich Anhaltspunkte von Gefährdungen ggf. dem Jugendamt zu melden.
} 
den Ausführungen von Vogd (2006, S. 225) innerhalb bestimmter Grenzen doch Freiheitsräume zur Verfügung stehen - die sicherlich ein hohes Maß an „Selbstaufopferung“ abverlangen, - ,sich in die Sphären einzuschalten, die außerhalb der Spezialkompetenzen liegen, für die er eingesetzt wird“. Der medizinische Kinderschutz ist damit ein interessanter Fall bei Überlegungen zu Fragen zum Verhältnis von Organisation und professioneller Arbeit (Klatetzki und Tacke 2005).

Mit meinen Ausführungen sollten Wandlungsprozesse medizinischer Praktiken der Gewalterkennung und deren zugrundeliegende Gewaltverständnisse sowie ihre soziologischen Nebenwirkungen in den Blick genommen werden. Meines Erachtens nach kann eine sozialwissenschaftliche Gewaltforschung, wenn sie sich diesen soziotechnischen Praktiken der Gewalterkennung und -diagnostik widmet, ohne einen eigenen, genuinen Gewaltbegriff auskommen. Hitzler (1999) hat vorgeschlagen, analytisch zwischen zwei Betrachtungsweisen von Gewalt zu unterscheiden: Eine handlungstheoretische Beschäftigung mit Gewalt rückt Gewalt-Tätigkeit in den Fokus, während eine definitionstheoretische Perspektive Gewalt-Erfahrungen betrachtet. Mit dem Begriff der Gewalt-Tätigkeit zielt Hitzler (1999, S. 13) auf „Gewalt intendiertes Handeln“, und unter Gewalt-Erfahrung versteht er zum einen, „wie ein Widerfahrnis als Gewalt definiert wird“ und zum anderen, wie „ein Akteur etikettiert wird als (intendiertermaßen) Gewalt ausübend“. Beide analytischen Perspektiven auf Gewalt können jeweils unabhängig voneinander verfolgt werden und schließen sich gewissermaßen gegenseitig aus. Hitzler (1999, S. 12) zufolge kann „Gewalt handlungstheoretisch nur aus der Täter-Perspektive verstanden und erklärt““ und andererseits „Gewalt aus der Opfer- und aus der Zuschauer-Perspektive nur etikettierungs- bzw. definitionstheoretisch“" rekonstruiert werden. Diese analytische Konzeption von Gewalt durch Hitzler weiter auszubauen und stärker zu berücksichtigen, könnte meines Erachtens produktiv für eine soziologische Gewaltforschung sein.

Während von Trotha (1997, S. 10) noch feststellte, dass „Gewalt ein analytisches Stiefkind der allgemeinen soziologischen Theorie“" ist, zeigt z.B. die Sammelbesprechung von Hauffe und Hoebel (2017) zur soziologischen Gewaltforschung eindrücklich, dass dem nicht mehr so ist. Nicht nur beim Lesen dieser informativen Übersicht zur gegenwärtigen Forschung zum Thema von Gewalt drängt sich die Annahme auf, dass die sozialwissenschaftliche Beschäftigung mit Gewalt zugleich voranschreitet und auf der Stelle tritt. Bezugnehmend auf den Vorschlag von Hitzler (1999) zeigt sich, dass in den meisten besprochenen Texten - und darüber hinaus oftmals beide (handlungstheoretische und definitionstheoretische) Perspektiven miteinander vermischt werden (Peters 2016, S. 111 f., Fußnote). Diese Vermischung beider Perspektiven wird selten reflektiert. ${ }^{8}$ Eine Weiterentwicklung dieser eher konträr zur gängigen Gewaltforschung liegenden sog. definitionstheoretischen Perspektive bringt eine allgemeine Gewaltsoziologie vielleicht nicht unmittelbar weiter, eröffnet aber zum einen Zugänge zu dem Phänomen Gewalt, die bisher kaum genutzt

\footnotetext{
${ }^{8}$ Gleichwohl gibt es Ausnahmen wie z. B. die Aufsätze von Schäfer und Dellwing (2016) oder Unterkofler (2016) aus einer ethnografischen Perspektive und die Beiträge von Peters (1995, 2016), die dezidiert definitionstheoretisch ausgerichtet sind. Diese Art und Weise der Auseinandersetzung mit dem Thema der Gewalt ist aber wesentlich seltener anzutreffen als handlungstheoretisch ausgerichtete Analysen.
} 
wurden. Zum anderen könnte dieser Ansatz ein Korrektiv sein, „der die Gewaltforschung davon abhalten [kann], allzu unvorbereitet in begriffsstrategische Fallen zu laufen“ (Liell 1999, S. 44). Gleichzeitig kann eine handlungstheoretische Auseinandersetzung mit Gewalt die andere Perspektive davor bewahren, dass diese ,,Gewalt“ relativistisch als bloßes Begriffsspiel“" (Liell 1999, S. 51) auflöst. Eine Weiterentwicklung der sog. definitionstheoretischen Perspektive muss nicht vorrangig mit einem etikettierungstheoretischen Ansatz arbeiten, wie verschiedene Untersuchungen aufzeigen (Liell 1999; Schäfer und Dellwing 2016; Unterkoffler 2016).

Eine stärkere analytische Berücksichtigung beider Perspektiven könnte weiterhin dazu beitragen, was Hauffe und Hoebel (2017) aufgrund ihrer Analyse der Dynamiken der gegenwärtigen Gewaltforschung für eine zukünftige Ausrichtung vorgeschlagen haben. Das ist zum einen ,eine breitere Auseinandersetzung mit zentralen Positionen zur Gewalt“ und auf der anderen Seite ,die gegenwärtige Fixierung auf die Collins'sche Mikrosoziologie aufzubrechen und gleichzeitig die möglichst detaillierte empirische Durchdringung konkreter Gewaltsituationen beizubehalten.“ (Hauffe und Hoebel 2017, S. 383) Für die direkte Analyse von Gewaltsituationen eignet sich ein definitionstheoretischer Ansatz nicht, aber z. B. für die Frage, was und wie überhaupt etwas als eine „,konkrete Gewaltsituation“ angesehen wird. Zu einer breiten Auseinandersetzung mit Positionen zur Gewalt kann dieser Ansatz produktiv eingesetzt werden, wie ich hoffentlich anhand meines Beitrages am Beispiel des medizinischen Kinderschutzes aufgezeigt habe.

Funding Open Access funding provided by Projekt DEAL.

Open Access Dieser Artikel wird unter der Creative Commons Namensnennung 4.0 International Lizenz veröffentlicht, welche die Nutzung, Vervielfältigung, Bearbeitung, Verbreitung und Wiedergabe in jeglichem Medium und Format erlaubt, sofern Sie den/die ursprünglichen Autor(en) und die Quelle ordnungsgemäß nennen, einen Link zur Creative Commons Lizenz beifügen und angeben, ob Änderungen vorgenommen wurden.

Die in diesem Artikel enthaltenen Bilder und sonstiges Drittmaterial unterliegen ebenfalls der genannten Creative Commons Lizenz, sofern sich aus der Abbildungslegende nichts anderes ergibt. Sofern das betreffende Material nicht unter der genannten Creative Commons Lizenz steht und die betreffende Handlung nicht nach gesetzlichen Vorschriften erlaubt ist, ist für die oben aufgeführten Weiterverwendungen des Materials die Einwilligung des jeweiligen Rechteinhabers einzuholen.

Weitere Details zur Lizenz entnehmen Sie bitte der Lizenzinformation auf http://creativecommons.org/ licenses/by/4.0/deed.de.

\section{Literatur}

Alberth, Lars, Doris Bühler-Niederberger, und Steffen Eisentraut. 2014. Wo bleiben die Kinder im Kinderschutz? Die Logik der Intervention bei Sozialarbeitern, Ärzten und Hebammen. In Kinderschutz. Wie kindzentriert sind Programme, Praktiken, Perspektiven?, Hrsg. Doris Bühler-Niederberger, Lars Alberth, und Steffen Eisentraut, 26-61. Weinheim: Beltz Juventa.

Apelt, Maja, und Konstanze Senge. 2015. Organisation und Unsicherheit - eine Einführung. In Organisation und Unsicherheit, Hrsg. Maja Apelt, Konstanze Senge, 1-13. Wiesbaden: Springer VS.

Bernd, Hermann, Reinhard Dettmeyer, Sibylle Banaschak, und Ute Thyen. 2016. Kindesmisshandlung: Medizinische Diagnostik, Intervention und rechtliche Grundlagen. Berlin, Heidelberg: Springer.

von Bismarck, Sylvester. 2013. Der praktische Umgang mit Kindesmisshandlung aus Sicht eines Mediziners. In Kindesvernachlässigung und Kindesmisshandlung. Medizinische und juristische Perspek- 
tiven, Hrsg. Matthias Lammel, Stephan Sutarski, Steffen Lau, und Michael Bauer, 29-38. Berlin: Medizinisch Wissenschaftliche Verlagsgesellschaft.

Bode, Ingo, und Hannu Turba. 2014. Organisierter Kinderschutz in Deutschland: Strukturdynamiken und Modernisierungsparadoxien. Wiesbaden: Springer VS.

Bode, Ingo, und Werner Vogd. 2016. Einleitung. Mutationen des Krankenhauses. In Mutationen des Krankenhauses. Soziologische Diagnosen in organisations-gesellschaftstheoretischer Perspektive, Hrsg. Ingo Bode, Werner Vogd, 1-25. Wiesbaden: Springer VS.

Burri, Regula Valérie, und Joseph Dumit. 2007. Introduction. In Biomedicine as culture. Instrumental practices, technoscientific knowledge, and new modes of life, Hrsg. Regula Valérie Burri, Joseph Dumit, 1-14. New York: Routledge.

Conrad, Peter, und Joseph W. Schneider. 1980. Deviance and medicalization. From badness to sickness. St. Louis: Mosby.

Debertin, Anette, und Sibylle Banaschak. 2013. Sexueller Missbrauch von Kindern aus medizinischer Sicht. Kindesmisshandlung und-vernachlässigung 16:4-11.

Eberl, Robert, Andrea Huber-Zeyringer, N. Curcic, und Michael Höllwarth. 2010. Die nicht akzidentelle Verletzung des Kindes und deren Erscheinungsformen. Der Chirurg 81:139-142.

Engfer, Anette. 1986. Kindesmisshandlung. Ursachen, Auswirkungen, Hilfen. Stuttgart: Enke.

Franzheld, Tobias. 2017. Verdachtsarbeit im Kinderschutz. Eine berufsbezogene Vergleichsstudie. Wiesbaden: Springer VS.

Gelles, J. Richard. 1976. Demythologizing child abuse. The Family Coordinator 25:135-141.

Görgen, Arno, Maria Griemmert, und Heiner Fangerau. 2013. Kindheit und Trauma. Medikalisierung und Skandalisierung im Umgang mit der Gewalt gegen Kinder. Trauma \& Gewalt 3:218-229.

Hacking, Ian. 1991. The making and molding of child abuse. Critical Inquiry 17:253-288.

Hacking, Ian. 1999. The social construction of what? Cambridge: Harvard University Press.

Hauffe, Tobias, und Thomas Hoebel. 2017. Dynamiken soziologischer Gewaltforschung. Soziologische Revue 40:369-384.

Helfer, E. Ray. 1978. Die Verantwortung und Rolle des Arztes. In Das geschlagene Kind, Hrsg. Ray E. Helfer, C. Henry Kempe, 69-93. Frankfurt a.M.: Suhrkamp.

Hildenbrand, Bruno. 2014. Das KJHG und der Kinderschutz: Eine verpasste Professionalisierungschance der Sozialpädagogik. In Sozialpädagogik zwischen Staat und Familie, Hrsg. Birgit Bütow, Marion Pomey, Myriam Rutschmann, Clarissa Schär, und Tobias Studer, 175-202. Wiesbaden: Springer VS.

Hitzler, Roland. 1999. Gewalt als Tätigkeit. Vorschläge zu einer handlungstypologischen Begriffsklärung. In Ordnungen der Gewalt: Beiträge zu einer politischen Soziologie der Gewalt und des Krieges, Hrsg. Sighard Neckel, Michael Schwab-Trapp, 9-19. Wiesbaden: Springer.

Inthorn, Julia. 2012. Die Arbeit von Kinderschutzgruppen: Ergebnisse einer zweistufigen Untersuchung. In Kinderschutzgruppen in Österreich, Hrsg. Julia Inthorn, Maria Kletečka-Pulker, 41-76. Wien: Verlag Österreich.

Jud, Andreas, Ulrich Lips, und Markus A. Landolt. 2009. Characteristics associated with maltreatment types in children referred to a hospital protection team. European Journal of Pediatrics 169:173-180.

Kempe, Annie. 2013. Dr. C. Henry Kempe: a daughter's perspective. In C. Henry Kempe: a 50 year legacy to the field of child abuse and neglect, Hrsg. Richard D. Krugman, Jill E. Korbin, 7-12. New York: Springer.

Kempe, C. Henry. 1978. Sexual abuse, another hidden pediatric problem: the 1977C. Anderson Aldrich lecture. Pediatrics 62:382-389.

Kempe, C. Henry, Frederic N. Silverman, Brandt F. Steele, William Droegemueller, und Henry K. Silver. 1962. The battered-child syndrome. JAMA 181:17-24.

Klatetzki, Thomas, und Veronika Tacke (Hrsg.). 2005. Organisation und Profession. Wiesbaden: VS.

Liell, Christoph. 1999. Der Doppelcharakter von Gewalt: Diskursive Konstruktion und soziale Praxis. In Ordnungen der Gewalt: Beiträge zu einer politischen Soziologie der Gewalt und des Krieges, Hrsg. Sighard Neckel, Michael Schwab-Trapp, 33-54. Wiesbaden: Springer.

Lynch, Margaret A. 1985. Child abuse before Kempe: an historical literature review. Child Abuse \& Neglect 9:7-15.

Marks, Svenja, Julian Sehmer, und Werner Thole. 2018. Arbeitsauftrag „Gefährdungsbereich“. Befunde aus einem Transferprojekt im Kinderschutz. Sozial Extra 42:12-14.

Meadow, Roy. 1989. ABC of child abuse. Epidemiology. British Medical Journal 298:727-730.

Mertens, Birgit, und Sabine Pankofer. 2011. Kindesmisshandlung: körperliche Gewalt in der Familie. Paderborn: Schöningh UTB.

Peters, Helge. 1995. Da werden wir empfindlich. Zur Soziologie der Gewalt. In Jugend und Gewalt. Devianz und Kriminalität in Ost und West, Hrsg. Siegfried Lamnek, 25-36. Opladen: Leske + Budrich. 
Peters, Helge. 2016. Situationen der Gewalt unter definitionstheoretischer Perspektive. In Situationen der Gewalt, Hrsg. Claudia Equit, Axel Groenemeyer, und Holger Schmidt, 107-127. Weinheim: Beltz Juventa.

Pfohl, F. Stephen. 1977. The "discovery" of child abuse. Social Problems 24:310-323.

Reiner, Frank. 1989. Definitionen und Epidemiologie. In Kindesmisshandlung. Eine Orientierung für Ärzte, Juristen, Sozial- und Erziehungsberufe, Hrsg. Hermann Olbing, Klaus-Ditmar Bachmann, und Rudolf Gross, 18-25. Köln: Deutscher Ärzte-Verlag.

Schäfer, Maximilian, und Michael Dellwing. 2016. Gewalt zwischen den Bühnen. Ethnografische Rekonstruktionen körperlicher Konfliktsituationen. In Situationen der Gewalt, Hrsg. Claudia Equit, Axel Groenemeyer, und Holger Schmidt, 150-173. Weinheim: Beltz Juventa.

Schwier, F., P. Manjgo, und M. Kieslich. 2019. Neue Entwicklungen im medizinischen Kinderschutz. Monatsschrift Kinderheilkunde 167:856-867.

Söhner, Felicitas, Heiner Fangerau, und Arno Görgen. 2017. Der medizinische Diskurs um Kinderschutz und dessen historische Entwicklung. In Präventive Strategien zur Verhinderung sexuellen Missbrauchs in Pädagogischen Einrichtungen. Kindeswohl als kollektives Orientierungsmuster?, Hrsg. Heiner Fangerau, Alexander Bagattini, Jörg M. Fegert, Rudolf Tippelt, Willy Viehöver, und Ute Ziegenhain, 63-96. Weinheim: Beltz Juventa.

Thun-Hohenstein, Leonhard. 2006. Interdisciplinary child protection team work in a hospital setting. European Journal of Pediatrics 165:402-407.

Thun-Hohenstein, Leonard. 2016. Kinderschutzgruppen. In Forensische Arbeit mit Kindern und Jugendlichen: Praxishandbuch für die interdisziplinäre Zusammenarbeit: Psychologie - Medizin - Recht Sozialarbeit, Hrsg. Sabine Völkl-Kernstock, Christian Kienbacher, 115-124. Wien: Springer.

von Trotha, Trutz. 1997. Zur Soziologie der Gewalt. In Soziologie der Gewalt, Hrsg. Trutz von Trotha, 9-56. Opladen: Westdeutscher Verlag.

Turba, Hannu. 2018. Die Polizei im Kinderschutz. Zur Verarbeitung institutioneller Komplexität in hybriden Berufswelten. Wiesbaden: Springer VS.

Unterkofler, Ursula. 2016. Wo beginnt Gewalt? (Implizite) professionelle Setzungen zwischen Gewaltfreiheit, Gewaltrelevanz und Gewalttätigkeit in Situationen offener Jugendarbeit. In Situationen der Gewalt, Hrsg. Claudia Equit, Axel Groenemeyer, und Holger Schmidt, 130-149. Weinheim: Beltz Juventa.

Verocai, Elmar, Ingo Kitzelmann, Florian Juen, und Burkhard Simma. 2013. Evaluation einer Kinderschutzgruppe bei einem unabhängigen Kooperationspartner - Vorschläge für eine Optimierung der Vernetzung. Klinische Pädiatrie 225:234-238.

Vogd, Werner. 2006. Verändern sich die Handlungsorientierungen von Krankenhausärzten unter den organisatorischen und ökonomischen Rahmenbedingungen? Ergebnisse einer rekonstruktiven Längsschnittstudie. Sozialer Sinn 7:197-230.

Williams, Gertrude J. Rubin. 1983. Editorial. Child protection. A journey into history. Journal of Clinical Child Psychology 12:236-243.

Christoph Sucherdt hat an der FSU-Jena Soziologie im Bachelor und Master studiert. Seit 2016 ist er wissenschaftlicher Mitarbeiter an der Universität Kassel im Fachgebiet Sozialwissenschaftliche Methodologie qualitativ-rekonstruktiver Forschung des Institutes für Sozialwesen. Dort liegt der Schwerpunkt seiner Forschung im medizinischen Kinderschutz, wo er an einer Promotion zu diesem Thema arbeitet. 